\section{Jean Bullier}

*GLOSS AIRE*
- Colliculus supérieur : région
du mésencéphale dorsal qui reçoit
des projections des cellules gan-
glionnaires de la rétine.
- Pulvinar inférieur : noyau
thalamique impliqué dans le sys-
tème visuel.
- Corps genouillé latéral : relais
thalamique des voies visuelles; il
reçoit des projections des cellules
ganglionnaires de la rétine et
projette vers la région striée du
lobe occipital du cortex cérébral
(aire visuelle primaire).
• Aires préstriées: régions du
lobe occipital du cortex cérébral
considérées classique ment
comme des aires visuelles secon-
daires.

\section{ADRESSE}

J. Bullier: directeur de recherche au Cnrs. Inserm U. 94, 16, avenue du Doyen-Lépine, 69500 Bron, France.

\section{TIRÉS A PART}

J. Bullier.

$\mathrm{m} / \mathrm{s} n^{\circ} 8$ vol. 5 , octobre 89

\title{
Les deux systèmes visuels : résurgences multiples d'une même idée
}

Le système visuel peut être subdivisé en deux sous-ensembles fonctionnels, l'un responsable de la discrimination et de la reconnaissance des formes (vision focale) et l'autre de leurs relations spatiales dans le champ visuel (vision ambiante). Les voies nerveuses empruntées par ces deux subdivisions sont en grande partie distinctes et aboutissent chez les primates au cortex inférotemporal (vision focale) et pariétal (vision ambiante). Une version plus ancienne de cette subdivision, persistant chez les rongeurs (tel le hamster), attribue au colliculus supérieur un rôle dans la vision ambiante et au cortex visuel la responsabilité principale de la vision focale. Cette organisation semble n'avoir pas été totalement abandonnée chez les primates où des expériences démontrent que le colliculus supérieur contribue également à la voie pariétale, et donc à la vision ambiante.

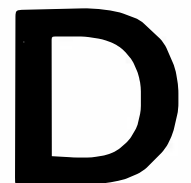

ivise et règne »; cette maxime s'applique aussi bien dans le domaine politique que dans celui de la recherche scientifique où l'un des premiers réflexes du chercheur confronté à un problème qui le dépasse est de le subdiviser. L'étude des mécanismes anatomo-physiologiques de la perception visuelle chez le mammifère constitue un bon exemple de cette approche. En effet, au cours des vingt dernières années, ce n'est pas moins de quatre fois qu'on a assisté à la division du système visuel du mammifère en deux sous-ensembles plus ou moins indépendants. Le but de cette synthèse est de faire le point sur ces différentes divisions, de présenter des résultats récents obtenus chez le singe macaque et de montrer comment le concept actuel de deux systèmes visuels englobe en quelque sorte la plupart des modèles proposés aupa- ravant. La première partie est une brève revue de la littérature qui présente de façon historique les différentes versions des deux systèmes visuels. Nous verrons ainsi que ces notions, bien que différentes, sont souvent confondues et que l'interprétation des résultats récents est grandement facilitée par cette perspective historique.

L'idée qu'il existe plusieurs sousensembles fonctionnels dans le système visuel n'est certes pas nouvelle puisque, d'après Polyak [1], la connaissance très rudimentaire de l'anatomie des voies visuelles à la fin du siècle dernier conduisait déjà von Monakow à distinguer trois systèmes, le premier associé au colliculus supérieur (ou tubercules quadrijumeaux antérieurs), le second au pulvinar et le troisième au corps genouillé latéral. Ces trois structures qui vont jouer un rôle important dans les différents modèles des deux systèmes visuels se retrouvent dans le 


\section{RÉFÉRENCES}

1. Polyak S. The vertebrate visual system. Chicago: University of Chicago Press, 1957.

2. Schneider. Two visual systems. Science $1969 ; 163: 895-902$.

3. Trevarthen C. Two mechanisms of vision in primate. Psychologisch Forschung 1968 ; 31 : 299-337.

4. Jeannerod M. Les deux mécanismes de la vision. La Recherche 1974 ; 5 : 23-32.

5. Goodale MA, Murrison RC. Effects of lesion of superior colliculus on locomotor orientation and the orienting reflex in the rat. Brain Res 1975 ; 88 : 243-61.

6. Holmes G. Disturbances of visual orientation. Br J Ophthalmol 1918; 2 : 449-86.

7. Mohler CW, Wurtz RH. Role of striate cortex and superior colliculus in visual guidance of saccadic eye movements in monkeys. J Neurophysiol 1977 ; 40 : 74-94.

8. Snyder M, Diamond IT. The organization and function of the visual cortex in the tree shrew. Brain Behav Evol 1968 ; 1 : 244-88.

9. Diamond IT. Organization of the visual cortex : comparative anatomical and behavioral studies. Fed Proc 1976 ; 35 : 60-7.

10. Schilder P, Pasik P, Pasik T. Extrageniculostriate vision in the monkey. III. Circle vs. triangle and « red vs. green » discrimination. Exp Brain Res 1972 ; 14 : 436-48.

11. Dineen J, Keating EG. The primate visual system after bilateral removal of striate cortex. Exp Brain Res 1981 ; 41 : 338-45.

12. Klüver H. Functional significance of geniculo-striate system. Biol Symposia 1942 ; 7 : 253-99.

13. Pöppel E, Held R, Frost D. Residual visual function after brain wounds involving the central visual pathways in man. Nature 1973 ; 243 : 295-6.

14. Weiskrantz L, Warrington EK, Sanclers MD, Marshall J. Visual capacity in the hemianopic field following a restricted corti-

schéma actuel des voies visuelles menant de la rétine au cortex occipital, pariétal et inférotemporal chez le singe macaque (figure 1). Selon ce schéma, les cellules ganglionnaires de la rétine se projettent sur le corps genouillé latéral dont la principale projection est l'aire 17, également appelée V1. L'aire 17 envoie des connexions sur le cortex préstrié qui entoure l'aire 17 dans le lobe occipital et qui lui-même se projette sur le cortex pariétal et le cortex inférotemporal. Une autre voie permet de relier la rétine au cortex visuel, c'est celle qui emprunte la connexion entre les cellules ganglionnaires de la rétine et le colliculus supérieur, celui-ci se projetant sur le noyau thalamique appelé pulvinar inférieur qui envoie des connexions sur le cortex visuel.

\section{Premier modèle}

Le schéma moderne des voies visuelles menant au cortex a pu être établi de façon progressive au cours des trente dernières années à l'aide de méthodes anatomiques et physiologiques et par l'étude du comportement visuellement guidé d'animaux ayant subi des lésions dans différentes régions du cerveau. On peut dire que le point de départ de la notion moderne de deux systèmes visuels se trouve dans un article célèbre de Schneider publié en 1969 [2] dans lequel il décrit les effets de lésions du colliculus supérieur et du cortex visuel sur le comportement visuellement guidé du hamster. Selon cet auteur, la lésion du colliculus supérieur provoque une incapacité de l'animal à s'orienter vers une cible présentée par l'expérimentateur (en l'occurrence une petite graine de tournesol), mais a peu d'effets sur la capacité de l'animal à apprendre une tâche de discrimination visuelle simple. En revanche, une lésion du cortex visuel rend l'animal inapte à un tel apprentissage mais n'a pas d'effet sur son comportement d'orientation. Ces résultats constituaient un bon exemple d'une double dissociation entre la localisation des lésions et les déficits induits, ce qui permit à Schneider de proposer une séparation entre deux systèmes visuels, l'un faisant intervenir le colliculus supérieur et permettant à l'animal de s'orienter visuellement dans son environnement, et l'autre, situé dans le cortex visuel et lui permettant d'apprendre à reconnaître visuellement la différence entre deux motifs géométriques simples. Plus schématiquement, selon Schneider, les deux questions « où ? » et «quoi ? » qui se posent dans l'identification d'un objet dans l'espace visuel sont traitées de façon parallèle, l'une par le colliculus supérieur et l'autre par le cortex.

La parution de cet article fut un événement dans le contexte de l'époque où les lésions du colliculus supérieur et du cortex chez les petits mammifères constituaient une des approches principales de l'étude du système visuel. A la suite d'études comportementales de la vision du primate, Trevarthen publia vers la même époque un article [3] qui eut également un impact certain sur la notion de deux systèmes visuels. Trevarthen faisait la distinction entre deux types de mécanismes distincts et complémentaires, la vision ambiante et la vision focale. La vision focale est celle qui intervient dans les tâches de manipulation d'objets et de reconnaissance de formes. C'est une vision dont le degré d'acuité est élevé, qui fait appel à la vision stéréoscopique et qui concerne principalement la partie centrale du champ visuel. La caractéristique principale de cette forme de vision est d'agir comme un pinceau de lumière balayant la scène visuelle au cours des mouvements des yeux et détaillant à chaque fixation une petite région du champ visuel. La vision ambiante, en revanche, capte l'étendue entière du champ visuel, possède un faible pouvoir de résolution spatiale mais est particulièrement sensible aux stimuli en mouvement et à la position des différents objets environnant l'individu, en particulier au cours de ses déplacements. Comme on pourrait s'en douter, Trevarthen situe la vision focale dans le cortex visuel et en particulier dans le lobe temporal et la vision ambiante dans le colliculus supérieur et la partie occipitopariétale du cortex. Nous verrons que, parmi tous les avocats des deux systèmes visuels, c'est celui qui avait vu le plus juste en ce qui concerne la localisation et la description de ces 


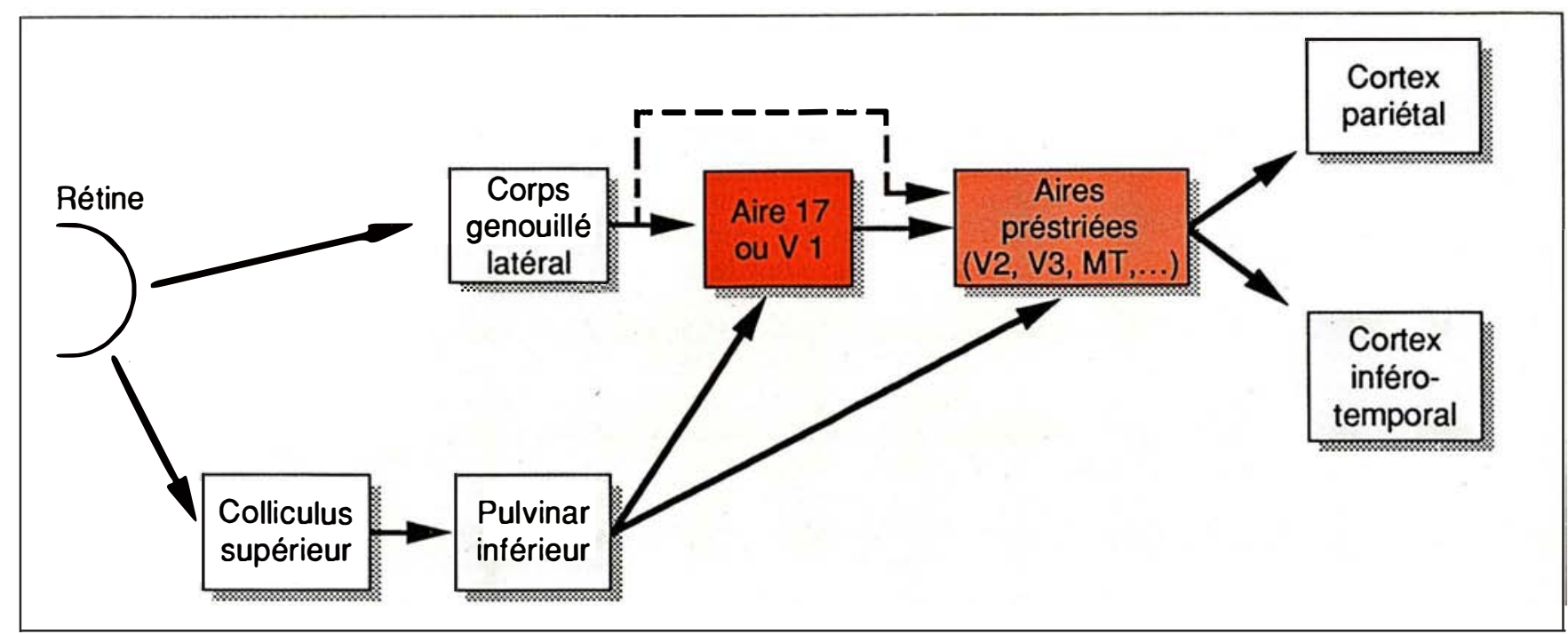

Figure 1. Schéma des voies visuelles reliant la rétine au cortex visuel chez le singe macaque. La ligne en pointillé reliant le corps genouillé latéral aux aires corticales préstriées représente une connexion beaucoup plus réduite que celle reliant le corps genouillé latéral et l'aire 17.

sous-ensembles. Les notions de vision ambiante et vision focale sont reprises par Jeannerod [4] qui met l'accent sur l'importance des mouvements des yeux qui relient en quelque sorte vision focale et vision ambiante. Il réalise une synthèse entre les points de vue de Trevarthen et Schneider en plaçant la vision focale dans le cortex visuel et en faisant du colliculus un noeud important dans le réseau de connexions impliquées dans les mouvements oculaires qui sont nécessaires pour intégrer vision focale et vision ambiante.

Malgré l'attrait exercé par ces concepts convergents concernant la présence de deux systèmes visuels parallèles situés dans le colliculus supérieur et le cortex, ce schéma ne résista pas au temps et aux tentatives infructueuses faites par d'autres chercheurs pour reproduire certains des résultats de Schneider [5]. S'il est généralement admis que le colliculus supérieur est impliqué dans les mécanismes d'orientation oculaire vers une cible, cet aspect du comportement peut également être perturbé par des lésions corticales, comme c'est le cas par exemple dans la paralysie oculomotrice ou «spasme de fixation » décrit par Gordon Holmes chez des patients atteints de lésions $\mathrm{m} / \mathrm{s} n^{\circ} 8 \mathrm{vol} .5$, octobre 89 du cortex pariétal [6]. Par ailleurs, on ne saurait dire que la notion de vision ambiante développée par Trevarthen se résume simplement à la capacité de réaliser des mouvements oculaires visuellement guidés, ce qui semble être une des fonctions essentielles du colliculus. Enfin, l'étude du comportement du singe macaque après lésion du colliculus supérieur et du cortex aboutit à des conclusions très éloignées des prédictions qu'on aurait pu faire au vu des résultats de Schneider chez le hamster. Par exemple, Mohler et Wurtz [7] montrèrent que la capacité de détecter une petite tache lumineuse dans le champ visuel du singe (la question « où ? ») et de faire une saccade dans cette direction n'est que légèrement affectée par une lésion du colliculus supérieur, mais qu'une lésion du cortex visuel entraîne également un déficit dans l'exécution de cette tâche.

\section{Autre version des deux systèmes visuels}

A la même époque que celle de la parution des travaux de Schneider et de Trevarthen, une seconde dichotomie du système visuel vit le jour à la suite des travaux de Diamond [8, 9] sur le comportement de discrimination visuelle chez le tupaïa, une espèce dont on pensait à l'époque que c'était un représentant des précurseurs des primates. Contrairement aux conclusions de Schneider, ces travaux mettaient en évidence le peu d'effet d'une ablation de l'aire 17 sur l'apprentissage de tâches de discrimination visuelle alors que la lésion additionnelle du colliculus supérieur ou du cortex visuel situé au-delà de l'aire 17 (le cortex visuel extrastrié) rendait l'animal totalement aveugle. Bien que désignés tous deux sous le même nom de deux systèmes visuels et souvent confondus entre eux, les concepts de Schneider et Diamond sont donc tout à fait différents. Pour Schneider, le colliculus supérieur et le cortex visuel jouent dans la vision des rôles différents et complémentaires, alors que pour Diamond il existe deux voies visuelles distinctes aboutissant au cortex extrastrié, la voie géniculo-striée passant par le corps genouillé latéral et l'aire 17 d'une part et la voie extragéniculée passant par le colliculus supérieur et le pulvinar d'autre part (figure 1). Selon Diamond, ces deux voies interviennent toutes deux dans la discrimination visuelle, la voie extragéniculée constituant la voie ancestrale, alors que la voie géniculo-striée serait une acquisition récente de l'évolution. 


\section{RÉFÉRENCES}

15. Perenin MT, Jeannerod M. Subcortical vision in man. Trends Neurosci 1979 ; 204-7.

16. Pohl W. Dissociation of spatial discrimination deficits following frontal and parietal lesions in monkeys. J Comp Physiol Psychol $1973 ; 82$ : 227-39.

17. Iwai E, Mishkin M. Further evidence on the locus of the visual area in the temporal lobe of the monkey. Exp Neurol 1969, 25 : 585-94.

18. Mishkin M. A memory system in the monkey. Philos Trans $R$ Soc London (Series B) $1982 ; 298: 85-95$.

19. Dean $P$. Visual behavior in monkeys with inferotemporal lesions. In : Ingle DJ, Goodale MA, Mansfield RJW, eds. Analysis of visual behavior. Cambridge: MIT Press, 1982 : 587-628.

20. Van Essen DC. Functional organization of primate visual cortex. In: Peters A Jones EG, eds. Cerebral cortex vol. 3 . New York: Plenum Publishing Corporation, 1985

21. Maunsell JHR, Newsome WT. Visual processing in monkey extrastriate cortex. Ann Rev Neurosci 1987 ; 10 : 363-401.

22. Bullier J. Cortical visual areas in the macaque monkey. In : Imbert M, ed. Models of visual perception : from natural to artificial. Oxford: University Press, 1989 (sous presse)

23. Von Bonin G, Bailey P. The neocortex of Macaca mulatta. Urbana: University of Illinois Press, 1947 : 165.

24. Morel A, Bullier J. Cortical connection of intraparietal and inferotemporal visual areas in the macaque monkey: a doublelabelling study. Soc Neurosci Abstr 1987, 177 : 14.

25. Ungerleider LG, Mishkin M. Two cortical visual systems. In: Ingle D J, Goodale MA, Mansfield RJW, eds. Analysis of visual behavior. Cambridge: MIT Press, 1982 : $549-86$

26. Rocha-Miranda CE, Bender DB Gross CG, Mishkin M. Visual activation of neurons in inferotemporal cortex depends on striate cortex and forebrain commissures.
A la suite de ces travaux, une grande partie des recherches des années 1970 s'intéressèrent à l'anatomie et à la physiologie des différentes étapes de la voie extragéniculée mais aboutirent à une impasse lorsqu'on compara les effets de lésions des deux voies parallèles chez le primate. En effet, il est clair que chez le singe comme chez l'homme, une lésion de la voie géniculo-striée donne lieu à des déficits visuels extrêmement importants et sans commune mesure avec ceux qui résultent de lésions du colliculus supérieur. Cette différence avec les résultats obtenus chez le hamster et le tupaïa peut en partie s'expliquer par le fait que, chez ces animaux, la quasi-totalité des fibres rétiniennes se projettent sur le colliculus supérieur et un tout petit contingent innerve le corps genouillé latéral, alors que c'est l'inverse chez le singe et l'homme.

Il apparaissait donc au début des années 1980 que, si on peut distinguer chez toutes les espèces deux voies d'accès de l'information visuelle au cortex, chez le primate la voie géniculo-striée est de loin la plus importante sur le plan fonctionnel alors que la voie extragéniculée ne joue qu'un rôle ancillaire. On ne peut s'empêcher cependant de voir une filiation directe entre les travaux de Diamond qui démontraient que la voie géniculo-striée n'est pas la seule à contribuer à la discrimination visuelle des formes et plusieurs travaux montrant sans ambiguïté qu'un singe ayant subi une ablation totale de l'aire 17 peut, à la suite d'un entraînement intensif, réaliser des tâches de discrimination visuelle [ 10 , 11]. Ainsi, contrairement aux conclusions de Klüver en 1942[12], qui constituèrent par la suite une sorte de dogme, il est maintenant admis que l'ablation de l'aire 17 ne produit pas une cécité totale et irréversible chez le singe macaque et que cette espèce ne constitue donc pas une exception dans le règne animal en ce qui concerne les effets de l'ablation de l'aire 17. Et l'homme dira-t-on? Si tous les animaux peuvent voir sans aire 17, pourquoi pas l'homme ? Ce n'est pas par hasard si l'idée selon laquelle l'ablation de l'aire 17 entraîne une cécité totale chez l'homme a été remise en question à peu près à la même époque que celle des travaux de Diamond sur les deux voies visuelles. Malgré un certain nombre de controverses, il est maintenant généralement accepté qu'il existe chez l'homme, après lésion de l'aire 17 , une forme de vision résiduelle inconsciente appelée blindsight [13, 14]. Connaissant l'importance donnée au colliculus supérieur par les travaux de Diamond sur la voie extragéniculée, on ne s'étonnera pas que la capacité résiduelle observée chez l'homme soit généralement attribuée au colliculus supérieur et à ses projections ascendantes [15].

\section{Troisième apparition des deux systèmes visuels}

Les deux systèmes visuels de Schneider ayant fait long feu et les deux voies visuelles de Diamond ayant abouti à une sorte d'impasse chez le primate, la notion de deux systèmes visuels va disparaître momentanément pour resurgir sous une autre forme au début des années 1980. Cette fois, c'est dans le lobe pariétal et le lobe temporal du singe macaque que sont traitées les deux questions « où ? » et « quoi ? » de la perception visuelle. Cette localisation fait suite aux travaux de Pohl [16], un élève de Mishkin, qui font apparaître une double dissociation entre les symplômes observés après ablation du cortex pariétal et du cortex temporal. Une brève description du dispositif expérimental et des résultats de Pohl nous aidera à comprendre ce qui est réellement démontré par ces travaux qui constituent le point de départ de toute une série d'études comportementales, anatomiques et physiologiques sur la division du travail entre lobe pariétal et lobe temporal chez le singe. Après avoir effectué une lésion corticale dans l'une ou l'autre de ces régions, on apprend à l'animal qu'une récompense est associée à l'un des deux objets qui lui sont présentés à des endroits variables sur un plateau (figure $2 A$ ). S'il se trompe, on lui montre son erreur en lui présentant seulement l'objet correct afin qu'il découvre la récompense qui lui est associée. Quand il a appris cette association entre un des deux objets et la récompense, on récompense le choix de l'autre objet. Initialement, l'animal fait de nombreuses erreurs mais il arrive à la 


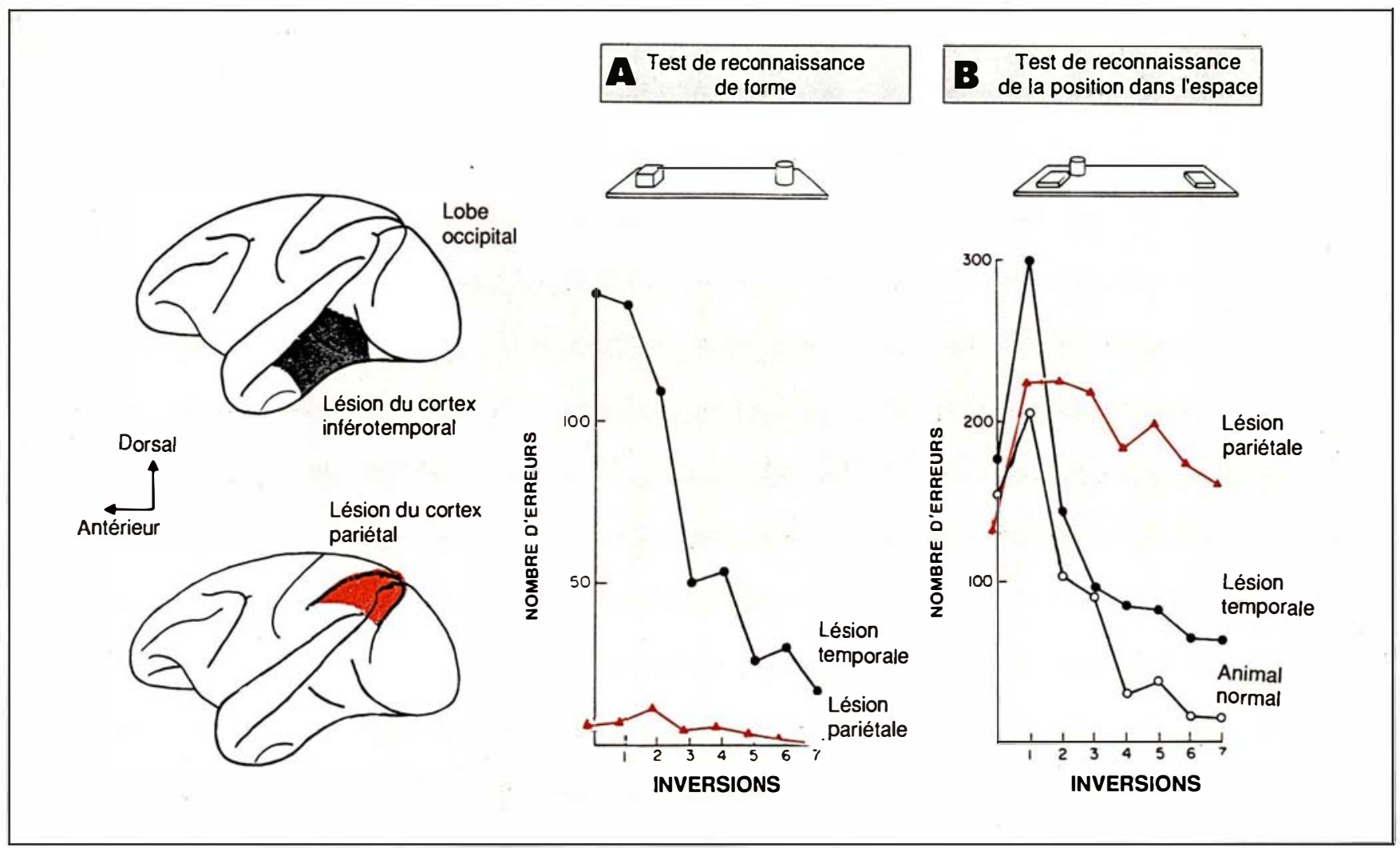

Figure 2. Expériences de Pohl démontrant le phénomène de double dissociation après lésion du cortex inférotemporal ou pariétal. La tache présentée en A est un test de discrimination visuelle dans lequel on apprend au singe à reconnaître un cube d'un cylindre. La courbe du nombre d'erreurs donne un aperçu des capacités résiduelles de l'animal ayant reçu une lésion. Après lésion du cortex inférotemporal, le nombre d'erreurs est élevé (courbe * lésion temporale $)$, alors qu'il est très faible après une lésion du cortex pariétal (courbe "lésion pariétale »). L'exercice présenté en B consiste pour l'animal à repérer la plaque qui se trouve la plus proche du cylindre repère. Après une lésion du cortex inférotemporal, l'animal fait un peu plus d'erreurs que l'animal normal, alors que le singe ayant reçu une lésion du cortex pariétal fait beaucoup d'erreurs. Ces résultats suggèrent que le cortex inférotemporal est impliqué dans la reconnaissance de formes alors que le cortex pariétal est important pour la vision des relations spatiales entre les objets dans le champ visuel.

longue à comprendre cette nouvelle association. A ce moment, on inverse de nouveau l'objet récompensé, et ainsi de suite. Au fur et à mesure de ces inversions successives, l'animal fait de moins en moins d'erreurs avant de reconnaître à coup sûr l'objet récompensé (par exemple courbe « lésion pariétale » sur la figure $2 A$ ). La courbe du nombre d'erreurs faites après chaque inversion avant identification correcte dépend, entre autres, des capacités de l'animal à reconnaître parmi les deux objets celui qui est récompensé. Cependant, il est clair que ce genre de test nécessite également de l'attention et de la mémoire et qu'on ne peut donc interpréter un déficit comme résultant d'une $\mathrm{m} / \mathrm{s} \mathrm{n}^{\circ} 8$ vol. 5 , octobre 89 atteinte spécifique de la reconnaissance et la mémorisation des formes que si le comportement général de l'animal n'est pas perturbé dans d'autres tests et si les performances de l'animal dans le test de reconnaissance de formes sont normales lorsque la lésion est placée dans une autre région du cerveau. C'est ce qui est observé dans le cas des expériences de Pohl quand les animaux sont testés pour leur capacité à reconnaître la relation spatiale entre des objets dans leur champ de préhension par la méthode suivante: on commence par apprendre à l'animal qu'il est récompensé s'il choisit la plaque située à côté du cylindre repère (figure $2 B$ ). Lorsqu'il ne fait plus d'erreurs on inverse et on récompense le choix de la plaque la plus éloignée du repère. Après cette inversion, l'animal fait un plus grand nombre d'erreurs avant de choisir à coup sûr la bonne plaque puis, après plusieurs inversions, il comprend le «truc», ce qui produit une forme caractéristique de courbe avec un pic suivi d'une décroissance rapide au cours des inversions successives (courbe « animal normal » sur la figure $2 B$ ).

Le phénomène de double dissociation est bien illustré sur la figure 2 : les animaux avec une lésion de la partie inférieure du lobe temporal (également appelé cortex inférotemporal) ont initialement beaucoup de 


\section{La quatrième version des deux systèmes visuels}

Une quatrième dichotomie entre deux ensembles fonctionnels au sein du système visuel a été récemment proposée [21, 30]. Cette fois, le point de départ se situe dans la rétine et le corps genouillé latéral. Danscette dernière structure en effet, on distingue deux groupes de neurones, les neurones des couches parvocellulaires, qui sont sensibles aux aspects chromatiques des stimuli mais possèdent une mauvaise sensibilité aux bas niveaux de contraste, et les neurones des couches magnocellulaires, qui sont insensibles à la couleur mais ont une excellente sensibilité aux faibles niveaux de contraste. Cette séparation en deux groupes de cellules dans le corps genouillé latéral se retrouve dans les aires V1 et V2, où on distingue deux voies circulant en parallèles, et dans les aires corticales $M T$ et $V 4$, qui sont respectivement sous l'influence des systèmes magnocellulaire et parvocellulaire. Cette division des aires corticales se superpose en quelque sorte à la division entre voie pariétale et voie inférotemporale, puisque le système magnocellulaire se projette principalement dans l'aire MT et est censé être impliqué dans la vision du mouvement et de la profondeur, qui sont plutôt des rôles attribués à la voie pariétale alors, que le système parvocellulaire est censé être utilisé dans la vision des formes et des couleurs, qui sont des tâches associées à la voie inférotemporale.

mal à apprendre le test de discrimination de formes puisqu'il leur faut trois jours et plus de cent erreurs pour arriver à repérer le bon objet. Après trois inversions, l'animal semble avoir compris et son nombre d'erreurs diminue bien qu'il reste plus important que celui d'un animal ayant reçu une lésion du cortex pariétal qui, lui, ne fait que très peu d'erreurs (figure $2 A$ ). Sur le test de reconnaissance de proximité du cylindre repère, les résultats sont inversés (figure $2 B$ ). Les animaux ayant subi une lésion du cortex inférotemporal (courbe «lésion temporale » sur la figure $2 B$ ) font un nombre d'erreurs seulement légèrement supérieur aux animaux contrôles alors que les animaux au cortex pariétal lésé semblent incapables de faire des progrès puisque leurs performances n'évoluent guère au cours des inversions successives (courbe « lésion pariétale » sur la figure $2 B$ ). Les résultats de Pohl ont été confirmés de nombreuses fois et sont à l'origine des associations entre vision du «quoi ? » et lobe inférotemporal et vision du « où ? » et lobe pariétal. La présentation des résultats originaux, bien que peut-être un peu fastidieuse, permet cependant de

du travail entre les deux systèmes visuels. Tout d'abord, la lésion pariétale diminue mais n'abolit pas les capacités de l'animal à apprécier les relations spatiales entre objets : l'animal arrive, après beaucoup d'erreurs, à reconnaître la bonne plaque. Ce qui semble surtout affecté, c'est sa capacité à apprendre l'association entre la plaque donnant lieu à une récompense et la distance par rapport au cylindre repère: après chaque inversion il fait autant d'erreurs qu'un animal naïf (figure $2 B$ ). De la même façon, un animal avec une lésion inférotemporale arrive éventuellement à reconnaître l'objet associé à la récompense. Autrement dit, il serait illusoire de conclure à une localisation absolue de la vision des formes dans le cortex temporal et de la vision de l'espace dans le lobe pariétal. La double dissociation entre les symptômes dus aux lésions pariétale et inférotemporale est en revanche bien établie. Il est clair que les animaux ayant reçu une lésion pariétale ne sont pas du tout diminués dans leur capacité à discriminer des formes (figure $2 A$ ). Le fait que les animaux à lésion inférotemporale soient légèrement affectés dans le test $B$ provient vraisemblablement du fait que ce test n'évalue pas seu- lement la capacité à apprécier les relations spatiales entre objets mais fait également intervenir la reconnaissance de formes.

Bien sûr, les résultats de Pohl ne faisaient que formaliser chez le singe une double dissociation déjà connue entre les symptômes résultant de lésions pariétales et temporales chez l'homme: chez celui-ci en effet, une lésion pariétale provoque de sérieux déficits dans la capacité qu'a l'individu d'appréhender visuellement les relations spatiales entre objets. situés dans l'espace environnant mais ne perturbe pas la reconnaissance des formes. Une lésion dans le lobe temporal, en revanche, n'affecte pas la perception de l'organisation spatiale de l'environnement visuel mais donne lieu à des déficits de reconnaissance de formes. Il est d'ailleurs usuel de distinguer l'agnosie visuelle provoquée par une lésion du cortex occipito-temporal et l'incapacité de saisir une scène visuelle dans son ensemble, ou simultanagnosie, que provoque une lésion bilatérale du cortex pariétal. La transposition au singe des résultats connus chez l'homme présente cependant deux avantages majeurs. Le premier est de pouvoir localiser avec précision le site exact des régions critiques dont la destruction provoque un déficit donné. C'est ainsi qu'à travers les travaux de Mishkin et ses collaborateurs, la localisation des régions critiques dans la reconnaissance et la mémorisation des formes a pu être progressivement établie. On sait par exemple que seules les lésions de la région inférotemporale provoquent des déficits de reconnaissance de formes. Il a en outre été possible de distinguer les conséquences de la destruction de la région antérieure du lobe temporal (aire TE sur la figure $3 A$ ), qui donne des déficits principalement mnésiques, de celles de lésions de la région intermédiaire entre le cortex préstrié et le cortex inférotemporal (aire TEO sur la figure $3 A$ ), qui semblent affecter principalement les capacités de catégorisation entre plusieurs objets [17-19].

Le deuxième avantage de la mise en évidence d'une double dissociation entre syndromes pariétal et temporal chez le singe est que l'organisation du système visuel est beaucoup mieux connue chez cet animal que 
chez l'homme et qu'il est à présent possible de suivre le cheminement de l'information visuelle entre l'aire 17 et le cortex pariétal et inférotemporal grâce à des travaux neurophysiologiques et neuro-anatomiques récents. Le cortex visuel du singe macaque a été subdivisé en plusieurs aires fonctionnelles dont la position sur l'écorce est présentée sur la figure 3 sur trois différentes vues, une latérale, une médiane et une supérieure. Les sillons, dont la position sur l'écorce est présentée sur chacune des petites vues du cerveau, ont été entrouverts afin de révéler les régions corticales qui y sont enfouies. De nombreuses revues sont consacrées à

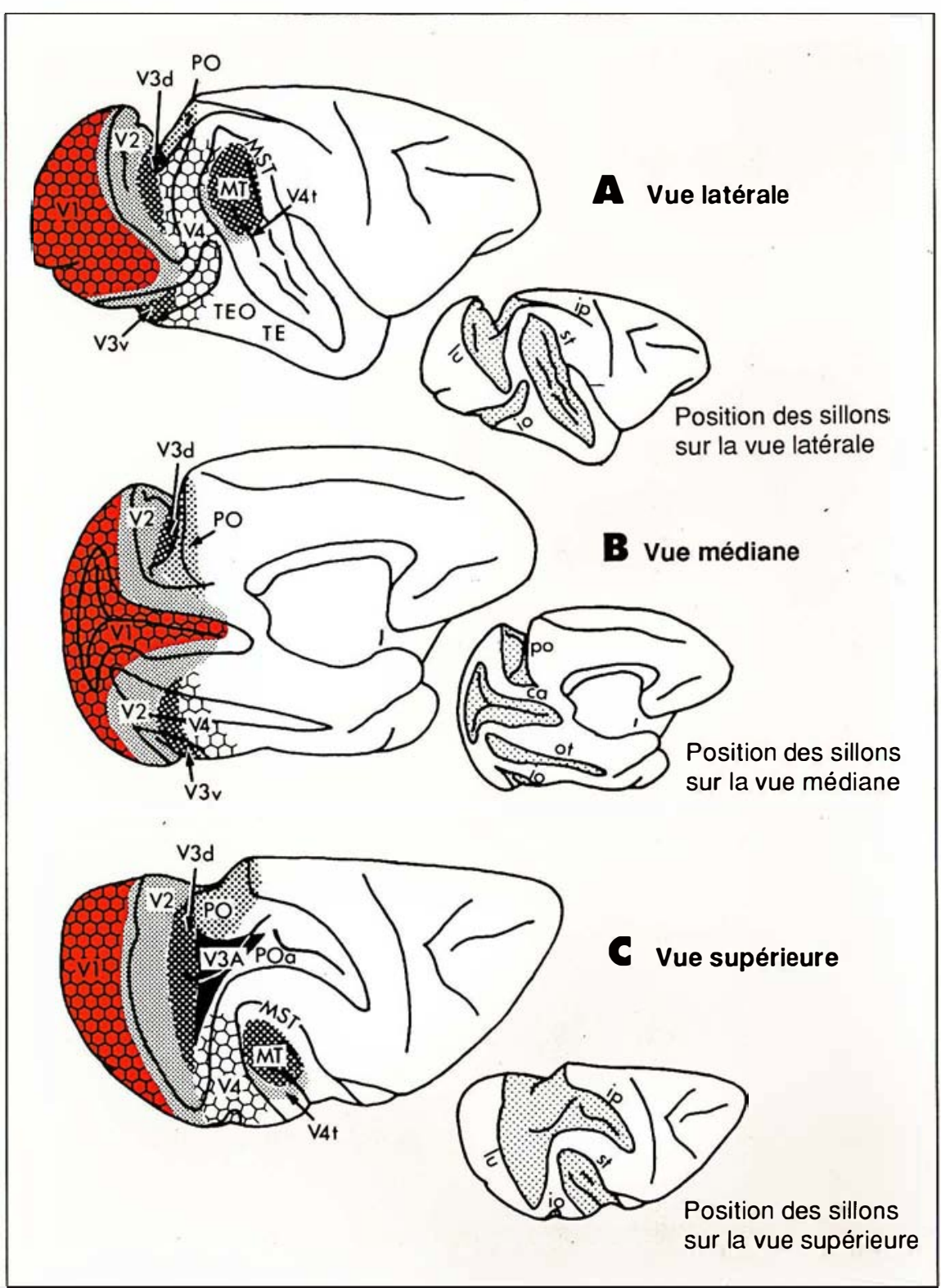

Figure 3. Aires corticales visuelles du singe macaque. A présente une vue latérale, $B$ une vue médiane et $C$ une vue supérieure du cortex. A chaque vue est associée une représentation plus réduite du cerveau qui illustre la position des sillons qui ont été entrouverts pour révéler les aires qui s'y trouvent cachées. ip : sillon intrapariétal; st: sillon temporal supérieur; lu : sillon lunaire; po: sillon pariéto-occipital; ca : fissure calcarine; ot : sillon occipito-temporal. la description des aires corticales visuelles du singe macaque et à leur rôle dans la vision [20-22] et seul l'essentiel sera rappelé ici. L'aire 17 , ou V1 sur la figure 3, occupe chez le singe la totalité de la région médiane enfouie dans la scissure calcarine et déborde assez largement sur la partie latérale de l'hémisphère cérébral. Entourant Vl sur pratiquement toute sa longueur se trouve l'aire V2, qui constitue seulement une petite partie de l'aire 18 de Brodmann mais correspond à l'aire $\mathrm{OB}$ de von Bonin et Bailey [23]. L'aire V3 est constituée de deux parties dénommées $V 3_{v}$ et $\mathrm{V} 3{ }_{\mathrm{d}}$ qui sont adjacentes à V2. En position rostrale par rapport à $\mathrm{V} 3$ on trouve l'aire V4, qui couvre une grande partie du cortex préstrié du singe, et l'aire MT, qui se trouve dans le fond du sillon temporal supérieur (st sur la figure 3) et qui est entourée par les aires V4, et MST. Enfin, il faut citer V3A qui se trouve enchâssée entre $V 3_{d}$, V4 et l'aire $P O$, qui se trouve dans la partie médiane du cortex préstrié (figures $3 B$ et $3 C$ ). Chacune de ces aires corticales contient une représentation plus ou moins complète de l'hémi-champ visuel controlatéral et est généralement séparée de ses voisines par des limites que l'on peut repérer sur des sections colorées de façon à révéler les corps cellulaires ou la myéline.

\section{Deux voies visuelles corticales}

L'information visuelle en provenance de la rétine est relayée par le corps genouillé latéral qui se projette presque exclusivement dans l'aire 17. De l'aire 17, l'information est principalement envoyée dans les aires V2, $\mathrm{V} 3{ }_{\mathrm{d}}$ et $\mathrm{MT}$ qui redistribuent cette information par un réseau très dense d'interconnexions entre les différentes aires corticales extrastriées. La question que nous nous sommes posée est de savoir par quels intermédiaires circule l'information visuelle qui aboutit au cortex pariétal et inférotemporal [24]. Nous étions en particulier intéressés à savoir si les mêmes aires corticales préstriées se projettent dans le cortex pariétal et inférotemporal ou bien si chacune de ces régions reçoit une information spécifique relayée par deux voies séparées dans le cortex préstrié. Pour 
cela, nous avons utilisé deux traceurs rétrogrades fluorescents différents que nous avons injectés, l'un dans l'aire TEO du cortex inférotemporal et l'autre dans l'aire POa du cortex pariétal (figure 3). Les résultats sont présentés dans la figure 4 sur des vues latérale et supérieure du cerveau de singe similaires à celles présentées sur la figure 3. Les régions corticales contenant des neurones se projetant dans TEO sont colorées en gris alors que celles contenant des neurones marqués par l'injection placée dans POa sont colorées en rose. Il est clair que les neurones afférents au cortex pariétal et inférotemporal occupent des régions presque entièrement séparées du cortex préstrié. Le cortex inférotemporal reçoit principalement de la région latérale du cortex préstrié et en particulier de l'aire V4, alors que le cortex pariétal est innervé principalement par des régions plus médianes comme l'aire $\mathrm{PO}$ ou des régions adjacentes à $\mathrm{MT}$

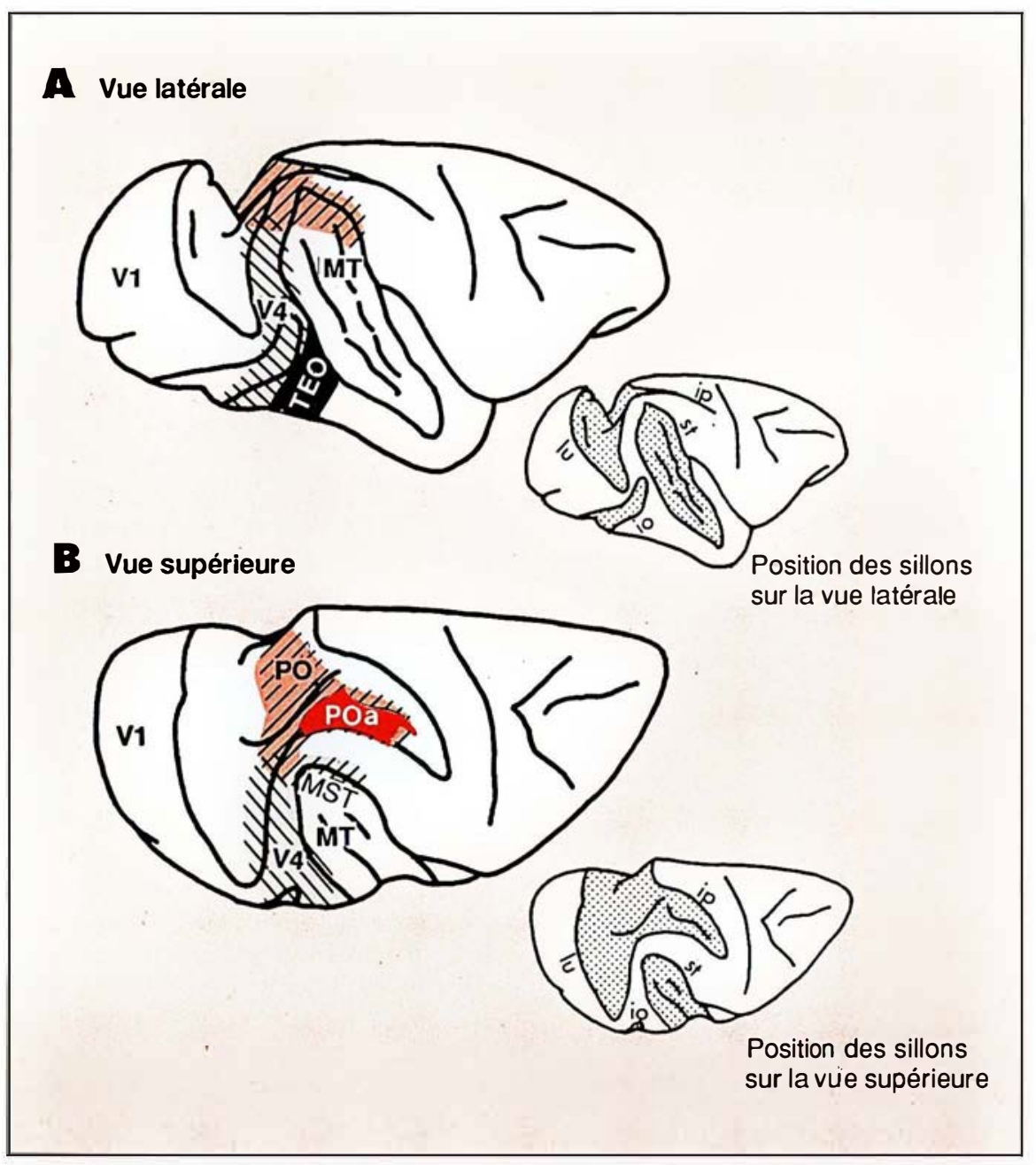

Figure 4. Régions corticales contenant des neurones marqués après des injections de traceurs rétrogrades fluorescents dans le cortex pariétal (aire POa) ou inférotemporal (aire TEO). Les régions contenant des neurones marqués par l'injection dans TEO sont colorées en gris alors que les régions contenant des neurones marqués par l'injection placée dans POa sont colorées en rose. II est clair que ces régions sont en grande partie séparées, les neurones se projetant dans TEO étant principalement dans les aires V4 et V3V, alors que les neurones afférents à $P O a$ appartiennent à MT, MST et PO. Une petite région située dans l'aire V3A se projette à la fois dans TEO et POa. dans le sillon temporal supérieur. Une petite région située au carrefour des aires V4 et V3A contient des neurones marqués par l'un ou l'autre des colorants et un petit pourcentage de neurones marqués à la fois par les deux traceurs.

Les résultats anatomiques présentés ci-dessus donnent une importance particulière au concept de deux voies visuelles corticales [25], l'une aboutissant au cortex inférotemporal et traitant de la vision des formes (vision focale) et l'autre aboutissant au cortex pariétal et traitant la vision de l'environnement (vision ambiante). Ce que l'on sait des propriétés des neurones situés dans les aires impliquées dans ces deux voies visuelles est en accord avec leur division fonctionnelle. Ainsi on sait que la région de l'aire $\mathrm{V} 4$ contenant des neurones se projetant sur TEO représente la partie centrale du champ visuel, ce qui s'accorde bien avec la notion de vision focale dans la voie inférotemporale. Par ailleurs, les neurones de l'aire V4 sont peu sélectifs pour les différents paramètres caractérisant le mouvement (direction, vitesse) des stimuli visuels alors qu'ils possèdent une forte sélectivité pour les aspects spatiaux (longueur, largeur, fréquence spatiale) et chromatiques des stimuli. Cette sélectivité est vraisemblablement utilisée lors de tâches de discrimination visuelle dans lesquelles la voie inférotemporale est impliquée. En ce qui concerne la voie pariétale, on trouve également une certaine adéquation entre le rôle supposé de cette voie dans la vision et les propriétés des neurones impliqués. Ainsi, la totalité du champ visuel est représentée dans les régions corticales contenant des neurones se projetant sur le cortex pariétal avec en particulier une forte représentation de la périphérie du champ visuel dans l'aire PO ; cela est en accord avec l'idée que la vision ambiante fait intervenir tout le champ visuel et en particulier la région périphérique. Enfin, les neurones des aires MT et MST qui font partie de la voie pariétale sont peu sensibles à la forme et à la couleur des stimuli visuels mais sont en revanche très sélectifs pour la direction du mouvement dans l'espace de ces stimuli, ce qui étaie l'idée selon laquelle la vision ambiante fait inter- 

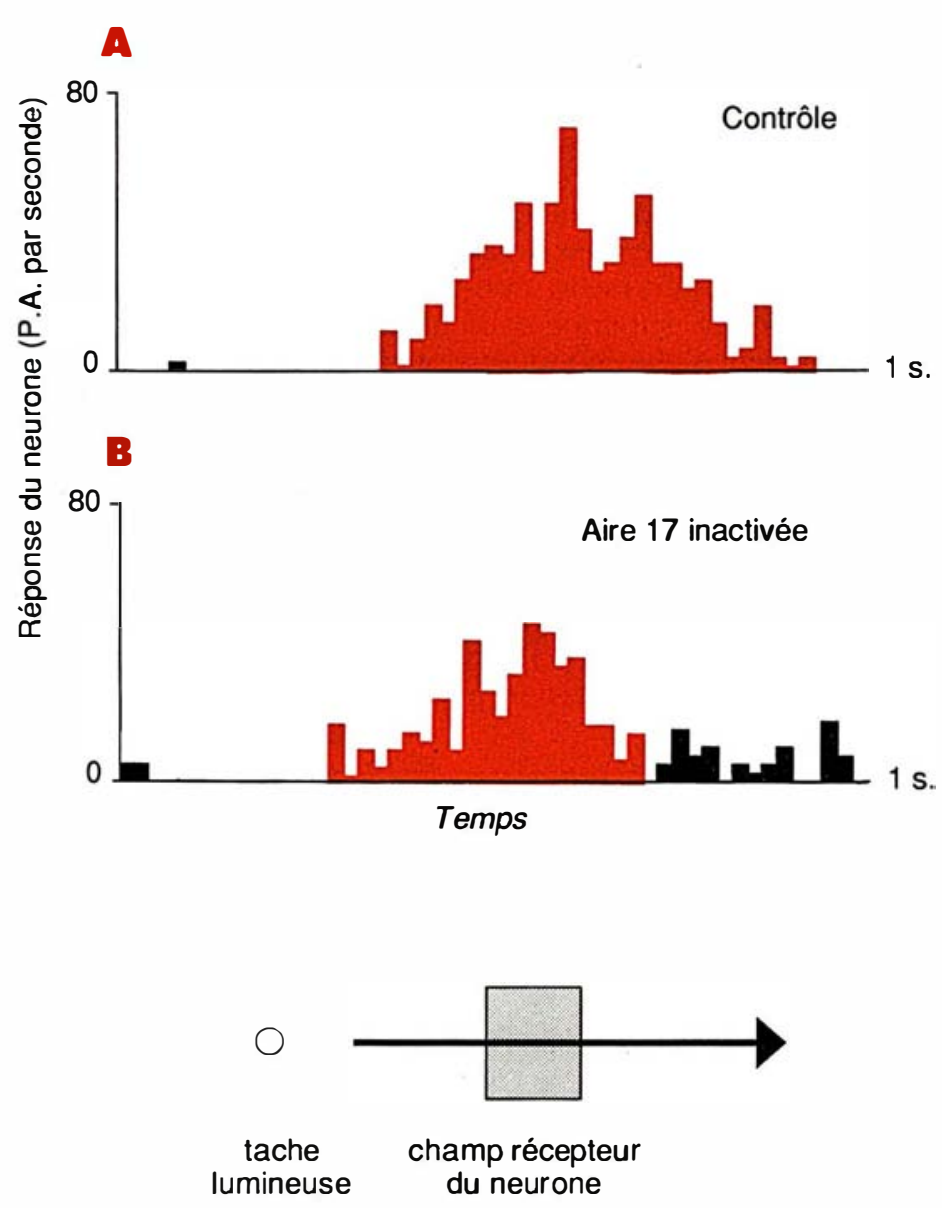

Figure 5. Réponse d'un neurone de l'aire MT à la stimulation par une tache lumineuse défilant dans son champ lécepteur. La réponse du neurone est représentée par la fréquence de décharge des potentiels d'action (PA). Lorsque la tache de lumière traverse son champ récepteur, cette activité augmente fortement (A). Lors de l'inactivation de V1 par le froid (B), cette réponse diminue mais n'est pas complètement abolie.

venir la perception du mouvement de l'image de l'espace environnant lors de déplacements de l'individu.

\section{La voie occipito-pariétale reste active après lésion de l'aire 17}

Une autre différence entre la voie inférotemporale et la voie pariétale a été récemment mise en évidence à la suite de lésions de l'aire 17 chez le singe. On savait depuis les travaux de Mishkin, Gross et leurs collaborateurs que l'ablation de l'aire 17 entraîne une perte des réponses visuelles des neurones dans l'aire dans son champ récepteur (A). Lors d'une inactivation par le froid de l'aire 17 , la réponse au même stimulus est réduite mais est néanmoins clairement présente (B). De tels neurones répondant à la stimulation visuelle ont été enregistrés dans MT lors d'inactivation réversible de l'aire 17 mais également quelques heures ou plusieurs semaines après lésion totale de cette aire [28].

Il ne fait donc pas de doute que la voie pariétale à laquelle appartient MT reste fonctionnelle lors de l'inactivation de l'aire V1, alors que la voie inférotemporale est totalement inopérante. Il est tentant dans ces conditions d'attribuer à la voie pariétale les capacités étonnantes de récupération de vision d'un singe, devenu fameux sous le nom d'Helen, ayant subi une lésion de l'aire 17 et dont le comportement a été remarquablement décrit dans un article de Humphrey [29]. Celui-ci montre en effet que la récupération fonctionnelle fait intervenir principalement la vision ambiante alors que la vision focale, et en particulier la reconnaissance de formes, demeure pratiquement inexistante chez cet animal. En allant plus loin, on pourrait également dire que la vision résiduelle chez l'homme après lésion de l'aire 17 fait intervenir la voie pariétale, et que le caractère inconscient de cette capacité résiduelle serait dû au fait que la voie inférotemporale est inactivée.

\section{Le colliculus supérieur revient sur la scène}

On pourrait croire que les voies pariétale et inférotemporale du cortex du primate nous ont entraîné bien loin de notre point de départ, les deux systèmes visuels de Schneider et le rôle du colliculus supérieur dans la vision ambiante. En fait il n'en est rien car le colliculus supérieur joue également un rôle important dans la voie pariétale chez le primate ainsi que le montrent des résultats récents de Gross et al. [30]. En effet, les neurones de l'aire MT deviennent silencieux à la suite de lésions combinées de l'aire 17 et du colliculus supérieur. Cela suggère donc qu'à la suite d'une lésion limitée à l'aire 17, l'activité résiduelle observée dans les neurones de la voie pariétale transite par le 
colliculus supérieur et que cette structure joue donc également un rôle important dans la vision ambiante chez le primate. Ainsi se trouve vérifiée une fois de plus cette loi bien connue de la biologie selon laquelle une stratégie n'est jamais complètement abandonnée au cours de l'évolution. Des deux systèmes visuels du hamster aux deux voies corticales du primate on trouve une continuité, celle de la participation du colliculus supérieur dans la vision ambiante. S'il est clair que l'importance accordée au colliculus supérieur est beaucoup moindre que celle qui lui était conférée par les travaux des années 1970, cette structure n'en demeure pas moins fortement associée à la voie pariétale et à la vision ambiante alors qu'elle semble peu impliquée dans la vision focale qui fait intervenir une voie largement indépendante conduisant de l'aire 17 au cortex inférotemporal

\section{Remerciements}

Je remercie plusieurs de mes collègues qui ont bien voulu lire et commenter ce texte. Leurs critiques ont permis de l'améliorer de façon sensible. Je remercie également Pascale Giroud pour son travail d'iconographie.

\section{RÉFÉRENCES}

27. Bullier J, Girard P. Visual responses of neurons in area V2 and in the superior temporal sulcus of the macaque monkey during reversible inactivation of area VI. Soc Neurosci Abst $1988 ; 243: 7$.

28. Rodman H, Gross CG, Albright TD. Afferent basis of visual response properties in area MT of the macaque. I. Effects of striate cortex removal. J Neurosci 1989 ; 9 : 2033-50.

29. Humphrey NK. Vision in a monkey without striate cortex : a case study. Percept $1974 ; 3: 241-55$.

30. Rodman HR, Gross CG, Albright TD. Responses of neurons in visual area MT after removal of the superior colliculus. Soc Neurosci Abst $1986 ; 372$ : 20.

31. Livingstone M, Hubel D. Segregation of form, color, movement and depth : anatomy, physiology and perception. Science 1989;

\section{Summary}

Two visual systems : multiple resurgences of the same idea

This paper reviews several attempts to segregate the mammalian visual system into two separate functional systems. Ancient versions of this model derived from studies on non-primate species allocated a role in ambiant vision to the superior colliculus and a role in focal vision to visual cortex, whereas the recent version in the monkey cortex suggests that ambiant vision is subserved by parietal cortex and that inferotemporal cortex is involved in focal vision. Pathways through the monkey prestriate cortex leading to the parietal and inferotemporal regions have recently been traced with double label retrograde tracing techniques. This demonstrates that the prestriate areas which project to each of these subdivisions are largely separate. It is also shown that most neurons in area MT remain active when area 17 is lesioned or silenced. This residual activity is apparently transfered through the superior colliculus which therefore plays also a role in ambiant vision in the primate visual system.

ERRATUM. Des problèmes techniques ont provoqué des erreurs de couleurs et de trames sur la figure 2 de l'article d'A. Rötig et al. : «Les remaniements du génome mitochondrial dans les déficits énergétiques de l'enfant » $\left(m / s n^{\circ} 7, v o l .5\right.$, p. 461, septembre 89). Nous la republions donc dans ce numéro.

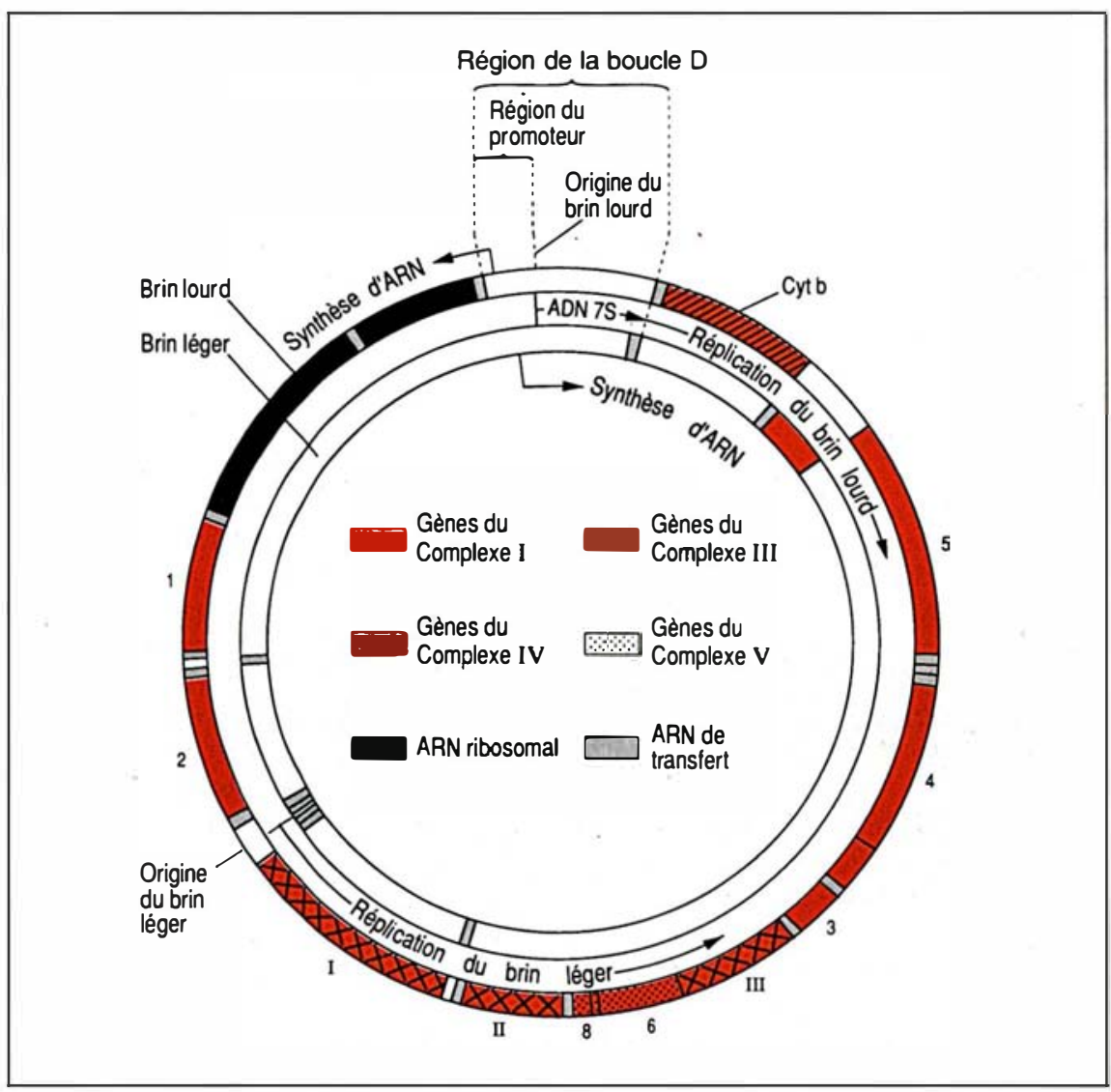

Figure 2. Organisation du génome mitochondrial. (D'après Wallace [39].) 